\title{
Clinical Effectiveness of Diode Laser as an Adjunct in the Treatment of Periodontitis
}

\author{
Bra'ah D Al Shbool', Rola A Habashneh ${ }^{2 *}$, Dafi S Ta'ani ${ }^{2}$ and Majdi M Alzoubi ${ }^{3}$ \\ ${ }^{1}$ Faculty of Graduate Studies, M Clin Dent in Dentistry/Periodontology, Jordan University of Science and Technology, \\ Jordan \\ ${ }^{2}$ Department Periodontology, Faculty of Dentistry, Jordan University of Science and Technology, Jordan \\ ${ }^{3} \mathrm{PH} . \mathrm{D}$ Researcher, Jordan
}

*Corresponding author: Prof. Rola Al Habashneh, Department of Preventive Dentistry, Faculty of Dentistry, Jordan University of Science \& Technology, P.O. Box (3030), Irbid 22110, Jordan

\begin{abstract}
Objective: This randomized clinical trial aimed at evaluation of the clinical effects of a 810-nm diode laser as an adjunct to scaling and root planing (SRP).

Methods: Twenty-eight patients with 37.5 years as mean age with periodontitis were selected for the split-mouth clinical study. Two treatment cohorts for SRP alone and for DL and SRP $(810 \mathrm{~nm}, 2 \mathrm{~W}$, pulsed mode $20 \mathrm{~Hz}$, for 20 seconds) were used for random assigning of patients through a split mouth design. Measurement of clinical parameters that included plaque index (PI), gingival index $(\mathrm{GI})$, clinical attachment loss (CAL), and pocket depth (PD) was undertaken at baseline and 1, 3 and 6 months after therapy.

Results: DL as an adjunct and SRP was used for treating 112 sites whereas SRP was used for treating 112 sites. Unlike baseline, all variables in each cohort showed significant improvements $(P<0.001)$ within 6-month followup. Regarding post-intervention, other parameters (PI, PD, $\mathrm{CAL})$ did not show a statistically significant variation $(\mathrm{P}>$ $0.05)$; however, the laser cohort had a significant reduction $(P<0.05)$ for $\mathrm{Gl}$.

Conclusion: GI improved significantly when DL was used an adjunct therapy alongside SRP as opposed to SRP only.

Clinical significance: Diode laser (DL) as the adjunct to SRP helps in control inflammation among periodontitis patients.
\end{abstract}

\section{Keywords}

Clinical parameters, Periodontitis, Diode laser, SRP

\section{Introduction}

Periodontitis refers to a chronic inflammatory disease that involves many factors and which is linked to dysbiotic plaque biofilms and progressively destroys tooth-supporting apparatus [1]. Periodontitis treatment could be achieved effectively using SRP through hand instruments such as curettes and scalers or sound devices [2] with locally or systemically prepared adjunctive therapy to SRP serving as complementary method [3]. Bone graft or surgical debridement without or with membranes is additional therapeutic modalities that can be considered [3].

In recent years, periodontal therapy has witnessed the wide application of light-amplified stimulated emission of radiation (LASER). Erbium-doped Yttrium Aluminium Garnet (Er:YAG) and Diode, Carbon Dioxide $\left(\mathrm{CO}_{2}\right)$, and Neodymium-doped Yttrium Aluminiu Garnet (Nd:YAG) constitute the main examples [4]. Out of these, treatment of periodontal pockets can be achieved using DL and Nd:YAG lasers as adjunctive therapy to SRP [4]. Although it is unable to ablate calculus [5] the bacterial effect that DL has on pathogens of periodontitis [6] presents numerous advantages than other lasers. In patients with aggressive periodontitis, some studies have demonstrated that $\mathrm{DL}$ as an adjunct of SRP led to an improvement in some clinical parameters [7]. Nevertheless, regarding gingival inflammation clinical parameters, some studies did not find positive results 
[8]. Unlike SRP, conflicting results have been found in numerous clinical trials that evaluated the clinical efficacy of DL $[7,8]$. Notably many studies that found significant differences in support of laser cohorts, there were questions regarding whether the results were clinically relevant, thus it was concluded that DL did not improve conventional treatment.

Regarding the treatment of patients with periodontitis, the available base of RCTs indicates that it is not evident that these adjunctive laser procedures improve the clinical benefits achieved by conventional strategies for mechanical debridement (SRP) [9].

Moritz, et al. have showed a significant reduction in both bacteria and inflammation when used diode laser of $805 \mathrm{~nm}$ as adjunct to SRP [6]. Other authors have also reported promising results in treatment of periodontitis or periimplantitis with the use of DL $[10,11]$. While few have not found additional benefit in the use of Gallium Arsenide laser adjunct to SRP [12], It is still not fully clear how the healing is enhanced in response to adjunctive use of lasers.

The data from elsewhere in the human body suggests that lasers can be effective tools in pain reduction, antiinflammation, and acceleration of wound healing [1315].

Lasers can be effective on oral microbial species and "disinfect" the periodontal environment $[6,16]$.

Salgam, et al. found that both SRP alone and SRP with an adjunct diode laser resulted in significant improvements in all clinical parameters after periodontal treatment. However, the whole-mouth clinical reduction was greater in the test group compared to the control group. In general, these changes were not accompanied with differences between groups suggesting that other mechanisms (e.g., bactericidal) in addition to the inflammation may regulate the wound healing process in response to laser therapy. One possibility is a localized impact on the gingival crevicular epithelium [17]. This was first suggested by Romanos, et al. in a pig model [18]. In their work, instrumentation of the soft periodontal tissues with a diode laser $(980 \mathrm{~nm})$ led to a complete epithelial removal in comparison to conventional treatment methods with hand instruments.

The results of a meta-analysis of Sgolastra, et al. demonstrated that the use of $\mathrm{DL}$ as adjunct to SRP did not provide any improvement in terms of clinical parameters [19]. These findings are consistent with those provided by a previous systematic review [4], which, however, only included one RCT with a short (3 months) follow-up time.

It has been suggested that conflicting results in the literature could be due to the lack of standardization of the reported irradiation parameters and to the inappropriate specification of dosimetry (power, beam area, time, dose, contact, or defocused irradiation mode). Future studies should be performed to specify the appropriate laser settings and dosimetry of this method [20].

\section{Methods}

\section{Study population}

This trial aimed to compare the clinical effect of using one episode of $D L$ as the adjunct to using conventional SRP alone in periodontitis treatment; this split-mouth designed single blinded randomized controlled clinical trial was undertaken at one center. The Institutional Research Board (IRB) at Jordan University of Science and Technology (JUST) approved the study. The revised Helsinki Declaration tenets were complied with by obtaining the written consent of participants and through provision of a detailed explanation of study methodology and purpose. All participants in the study were adult patients that consecutively attended JUST Dentistry School in Periodontology Department between February and August 2017.

Inclusion of patients within the study was based on several factors including: ability of compliance with three follow up visits, willingness to participate in DL therapy, female and male patients above 18 years (female 21, male 7), and healthy patients with periodontitis (Interdental CAL is detectable at $\geq 2$ non-adjacent teeth in two quadrants with each quadrant having a minimum of 4 sites of PD exceeding $4 \mathrm{~mm}$, all patients had coronal bone loss around teeth.

The excluded patients were aged below 18 years, had sites where teeth extraction was to be studied, had faulty teeth restoration featured in the disease, were currently undergoing or had been on antibiotic therapy for the past 6 months, were subjected to periodontal treatment in the past 6 months, were drug/alcohol addicts or smokers, were lactating mothers and pregnant women, had systemic disease, which complicated their therapy or had systemic diseases, which might impede healing of wounds (for instance, diabetes).

\section{Sample size}

A primary outcome variable, $0.80 \mathrm{CAL}$ power level alongside 0.40 effect size of $\alpha=0.050$ was considered while calculating the size of the sample. Each cohort will require a sample of 97 sites selected from 28 patients that enrolled in the study.

\section{Data collection}

One experienced operator treated all patients. An independent calibrated examiner who did not feature within the treatment recorded all parameters (B.D.SH). In all examinations, good reliability $(I C C=0.766)$ was found and Cohen's $\mathrm{k}$ coefficient that predicted good reliability degree $(0.855)$ was calculated using interexaminer calibration. The measurements derived from 
each separate examination were computed using the kappa coefficients.

\section{Clinical examination}

The examiner that was blinded to study cohorts examined the 28 patients that met the exclusion and inclusion criteria. Bleeding probing sites and plaque index were used for assessing periodontal status and oral hygiene of teeth [9]. Clinical attachment loss and probing depth of pockets were measured using standardized periodontal probes whereas gingival status and plaque accumulation was assessed using sterile explorers and dental mirrors.

Williams's marked Michigan O periodontal probes (Diatech, Switzerland) used for every tooth for measuring parameters at six sites namely distolingual, midlingual, mesiolingual, distofacial, midfacial, and mesiofacial. The means for clinical attachment level (CAL), probing depth $(P D)$, gingival index $(\mathrm{GI})$, and plaque index (PI) of all sites were determined.

For adequate control of plaque before baseline examination, all patients practiced the procedure for oral hygiene for 7 days.

During clinical evaluation, 8 deep pockets that met the criteria for inclusion were chosen from each patient, 2 (posterior interproximal sites each quadrant) from 28 patients, Therefore, the study included 224 dental sites (112 sites within each cohort). The sites within group I (laser group) received LD (quicklase quickwhite, United Kingdom $810 \mathrm{~nm}$, pulsed at $20 \mathrm{~Hz}$, total power of $2.0 \mathrm{~W}$ that 400 micron optic fiber, uninitiated tip delivered) applied for 20 seconds for every periodontal pocket, as one application besides SRP, while SRP alone was administered to group II (control group) in the same patient.

\section{Investigator masking and randomization: (Single blinded)}

To receive treatment modalities of both methods each quadrant of the selected pair of sites was randomly allocated to the control group (SRP) or in the test group (SRP with adjunctive laser therapy) by tossing a coin.

Periodontitis clinical parameters were evaluated upon follow-up appointments with the same examiner directly conducting the clinical examination, and assigned of the measurement was done on the basis of follow-up appointments.

\section{Treatment protocol}

A total of 224 sites with deep pocket selected from 28 systemically healthy patients diagnosed with periodontitis. After full medical, dental History and initial periodontal therapy; SRP were done for each patient local anesthesia (2\% lidocaine with adrenaline 1:100.000) using periodontal Gracey curettes, ultrasonic scalers and abrasive paste with a brush or a rubber cup. The sites were divided into two groups: $1^{\text {st }}$ group received (SRP) with DL as adjunctive therapy group DL (QuickLase QuickWhite, British manufacturing company, $810 \mathrm{~nm}$, pulsed at 10 $\mathrm{Hz}$, total power of $2.0 \mathrm{~W}$, delivered by 400 micron optic fiber) applied for 20 seconds to the periodontal pocket to remove the epithelium of the periodontal pockets and disinfect them. $2^{\text {nd }}$ group included sites in the contralateral site in the same patient and received SRP only, within 24 hours.

The clinical parameters were evaluated at the first visit before laser application and re-evaluated at 1, 3, 6 months.

\section{Data analysis}

The Statistical Package for Social Sciences (SPSS) software version 25.0 (SPSS ${ }^{\circ}$ : Inc., Chicago, IL, USA) was used for analyzed the collected data. Data normality was checked using the Shapiro-Wilk test. Calculation of standard deviations, means and percentages was undertaken. Additionally, the treated sites for SRP (control) and SRP + DL (test) in terms of clinical parametric variables $(\mathrm{PI}, \mathrm{Gl}, \mathrm{CAL}$, and $\mathrm{PD})$ at different duration were compared using the Wilcoxon signed ranks test. Additionally, for comparison of subgroups, Mann-whitney and Kruskal Wallis tests were used. The statistical significance level was set at $(P \leq 0.05)$. ANOVA was used for comparing variables between continuous variables.

\section{Result}

Overall, the analyses (Figure 1) featured 28 patients with the mean age at 37.5 years (ranging between 28 and 55 years). Table 1 shows the baseline characteristics for the 28 patients. Based on the table, most participants had attained the educational level of university or college, were below 40 years, and their gender was female.

\section{Baseline clinical parameters characteristics}

Similar characteristics were found for $\mathrm{PI}$ (control group: 1.88; test group: 1.86; $\mathrm{P}=0.80$ ), $\mathrm{Gl}$ (control group: 2.32; test group: 2.38; $\mathrm{P}=0.34$ ); $\mathrm{CAL}$ (control group: $3.13 \mathrm{~mm}$; test group: $3.35 \mathrm{~mm} ; \mathrm{P}=0.06$ ), and PD (control group: $4.93 \mathrm{~mm}$; test group: $5.03 \mathrm{~mm}$; P $=0.26)$ following baseline examination. The clinical parameters in the two comparative groups in terms of standard deviations and means are illustrated in Table 2. No statistical variations were found between the two treatment protocols utilised at baseline visits for all clinical parameters $(P>0.05)$.

\section{Changes in (PD) between test and control groups over time}

At control sites, PD had means of $2.70 \mathrm{~mm}, 2.84$, and 2.96) whereas at test sites the means were $(2.62,2.54$, 2.87) at 6, 3, and 1 months. In terms of PD measurement, treatment protocols for 6,3 , and 1 month appointment, no significant variations were found $(p=0.29,0.10$, 0.77) (Table 2 and Figure 2). 


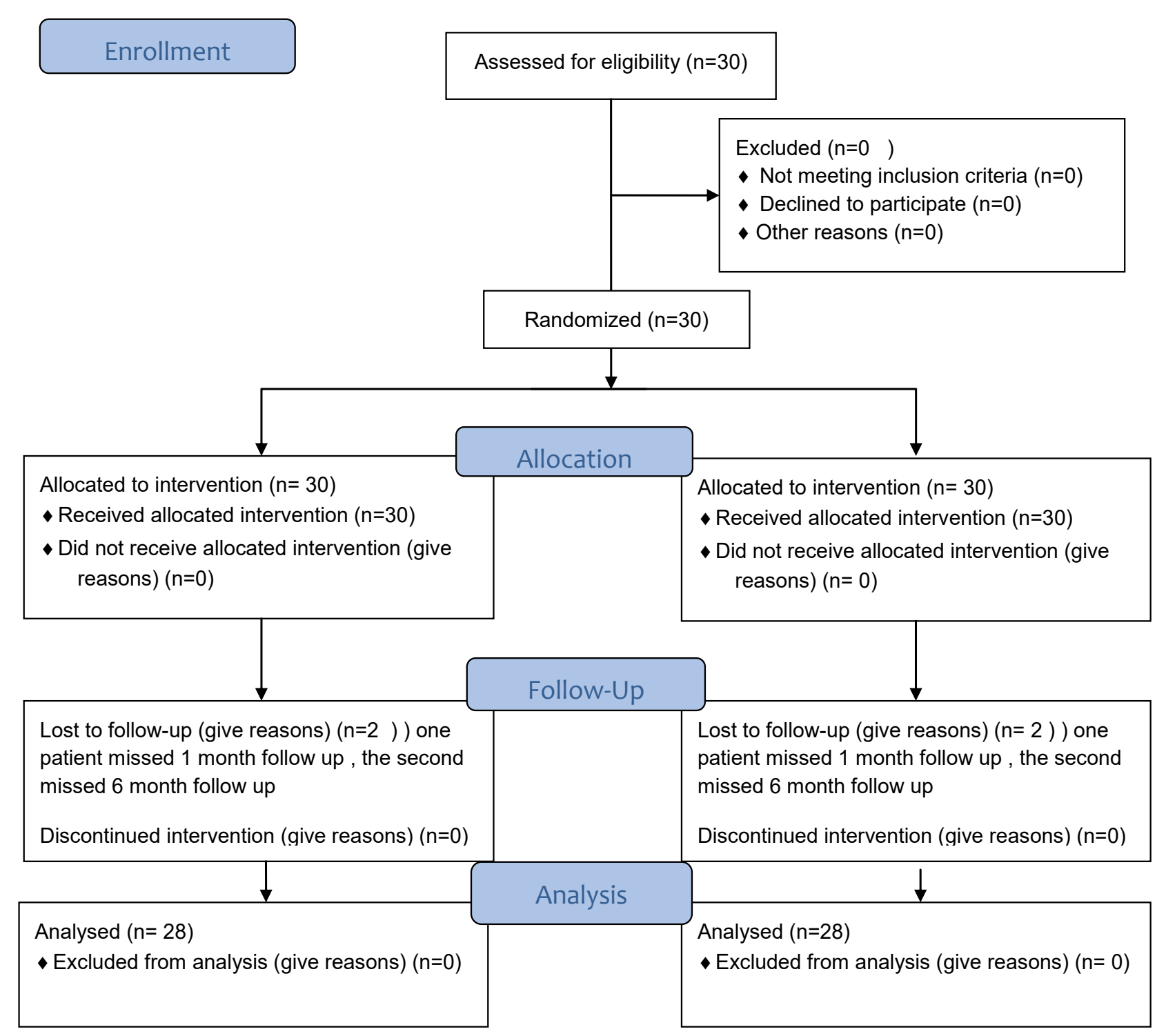

Figure 1: Flow diagram.

Table 1: Frequency distribution of demographic variable of study sample by patients and sites.

\begin{tabular}{|l|l|l|}
\hline Demographic Variable & $\begin{array}{l}\text { Patient No (\%) } \\
\text { (N = 28) }\end{array}$ & $\begin{array}{l}\text { Site No (\%) } \\
\text { (N = 224) }\end{array}$ \\
\hline Age & \multicolumn{2}{|l|}{} \\
\hline $20-40$ & $21(75.0 \%)$ & $168(75.0 \%)$ \\
\hline $41-60$ & $7(25.0 \%)$ & $56(25.0 \%)$ \\
\hline Gender & $10(35.7 \%)$ & $80(35.7 \%)$ \\
\hline Male & $18(64.3 \%)$ & $144(64.3 \%)$ \\
\hline Female & $14(50.0 \%)$ & $112(50.0 \%)$ \\
\hline Educational level & $14(50.0 \%)$ & $112(50.0 \%)$ \\
\hline Basic education & & \\
\hline $\begin{array}{l}\text { College/University } \\
\text { education }\end{array}$ & & \\
\hline
\end{tabular}

\section{Changes in (CAL) between test and control groups over time}

At control sites, the means of CAL for 6,3 , and 1 months were $1.19,1.13$, and 1.43 whereas at test sites the means were $1.07,1.00$, and 1.59 . In regard to CAL measurements, the two treatment protocols did not show significant difference at 6,3 , and 1 month appointments ( $p=0.28,0.42$, and 0.77 ) (Table 2 and Figure 3).

\section{Changes in GI between the test and control over time}

At control sites, the means of GI for 6,3 , and 1 month were $0.88,0.90$, and 1.08 whereas at test sites the means were $0.69,0.56$, and 0.81 . In regard to GI measurements, the two treatment protocols did not show significant difference at 6,3 , and 1 month appointments $(p=0.04$, $0.01,0.04$ ) (Table 2 and Figure 4).

\section{Changes in (PI) between test and control over time}

At control sites, the means of $\mathrm{PI}$ for 6,3 , and 1 months were $0.49,0.45$, and 0.46 whereas at test sites the means were $0.49,0.45$, and 0.46 . In regard to PI measurements, the two treatment protocols did not show significant difference at 6,3 , and 1 month appointments $(p=0.30$, 
Table 2: Means and standard deviations of studied clinical parameters at baseline and over.

\begin{tabular}{|c|c|c|c|c|c|}
\hline Variable & Baseline & 1-month & 3-month & 6-month & P-value \\
\hline \multicolumn{6}{|l|}{ PD (mm) } \\
\hline SRP + DL & $5.03(1.04)$ & $2.87(1.24)$ & $2.54(0.98)$ & $2.62(1.1)$ & $<0.001^{*}$ \\
\hline SRP & $4.93(0.99)$ & $2.96(1.2)$ & $2.84(1.04)$ & $2.70(1.01)$ & $<0.001^{*}$ \\
\hline P-value & 0.26 & 0.77 & 0.10 & 0.29 & \\
\hline \multicolumn{6}{|l|}{ CAL (mm) } \\
\hline SRP + DL & 3.35 (1.71) & $1.59(1.90)$ & 1.00 (1.59) & 1.07 (1.64) & $<0.001^{*}$ \\
\hline SRP & $3.13(1.87)$ & $1.43(1.67)$ & $1.13(1.68)$ & $1.19(1.78)$ & $<0.001^{*}$ \\
\hline$P$-value & 0.06 & 0.77 & 0.42 & 0.28 & \\
\hline \multicolumn{6}{|l|}{ GI } \\
\hline SRP + DL & $2.38(0.63)$ & $0.81(0.78)$ & $0.56(0.71)$ & $0.69(0.74)$ & $<0.001^{*}$ \\
\hline SRP & $2.32(0.63)$ & $1.08(0.86)$ & $0.90(0.92)$ & $0.88(0.91)$ & $<0.001^{*}$ \\
\hline P-value & 0.34 & 0.04 & 0.01 & 0.04 & \\
\hline \multicolumn{6}{|l|}{ PI } \\
\hline SRP + DL & $1.86(0.75)$ & $0.37(0.55)$ & $0.27(0.54)$ & $0.4(0.61)$ & $<0.001^{*}$ \\
\hline SRP & $1.88(0.71)$ & $0.46(0.67)$ & $0.45(0.63)$ & $0.49(0.62)$ & $<0.001^{*}$ \\
\hline P-value & 0.80 & 0.44 & 0.09 & 0.30 & \\
\hline
\end{tabular}

PD: Pocket Depth; CAL: Clinical Attachment Loss; GI: Gingival Index; PI: Plaque Index

\section{changes in PD between test and control over time}

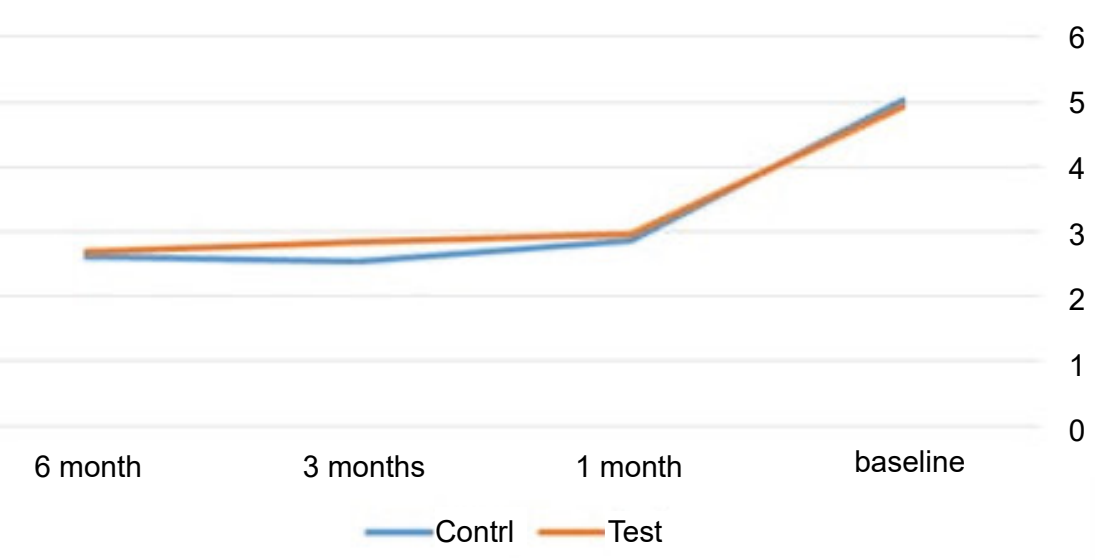

Figure 2: The changes in PD means in both groups over time.

\section{changes in CAL between test and control over time}

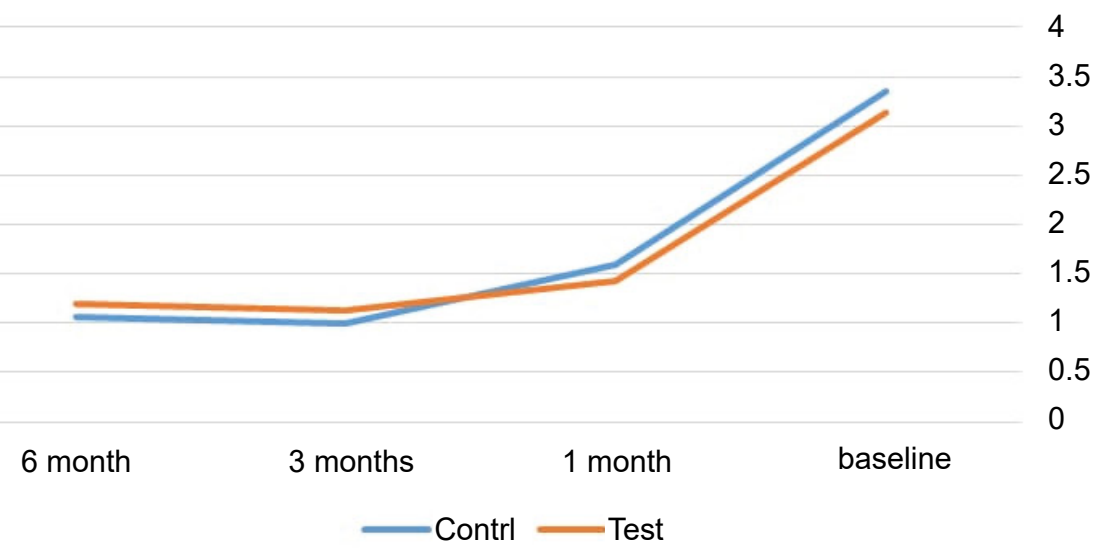

Figure 3: The changes in CAL means in both groups over time. 


\section{changes in GI between test and control over time}

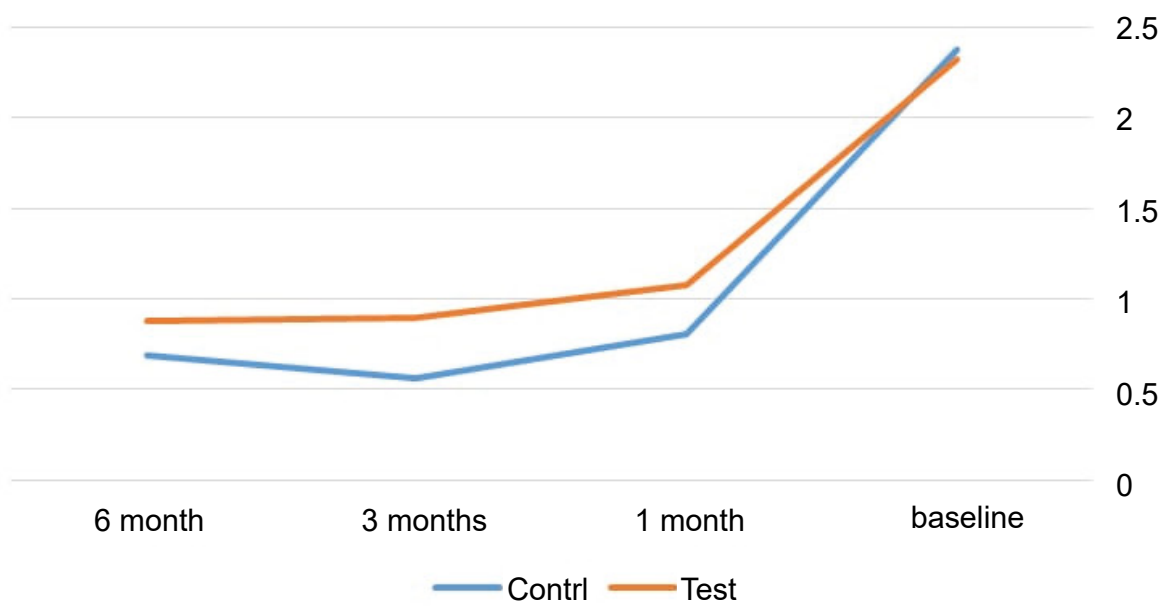

Figure 4: The mean difference of $\mathrm{Gl}$ between the test and control over time.

\section{changes in PI between test and control over time}

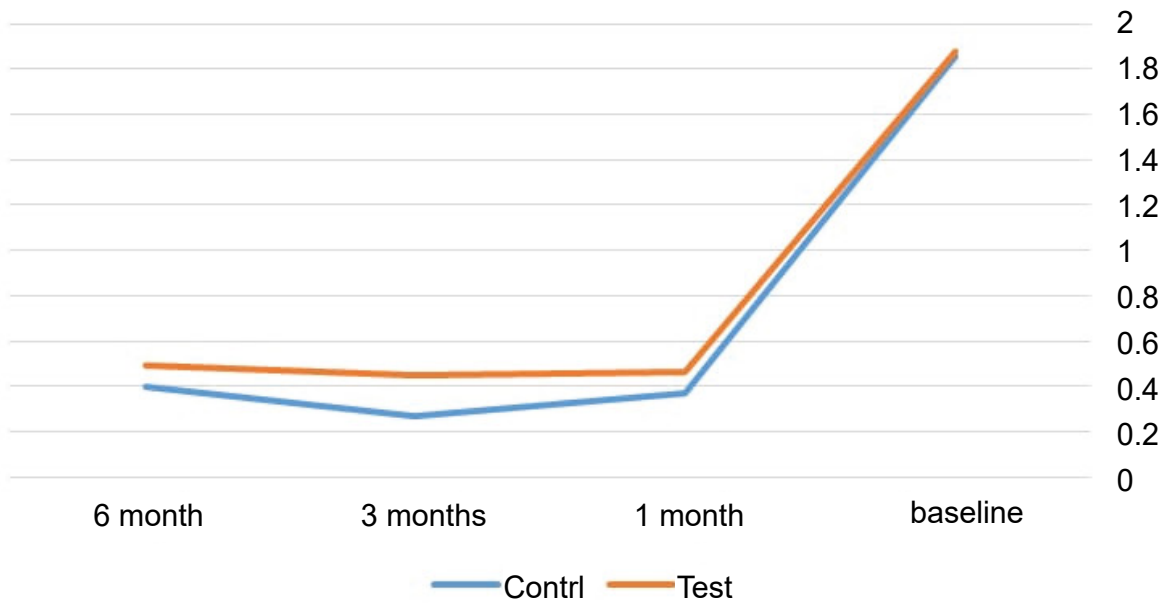

Figure 5: The mean difference of PI between the test and control over time.

\subsection{9, 0.44) (Table 2 and Figure 5).}

\section{Discussion}

Several dentistry fields use laser and in most studies it constituted the subject [21]. In this study, there were no complications (for instance, ulcers, abscess, or infection) as all postoperative healing cases were uneventful. After periodontal treatment, there were significant improvements in clinical parameters for the two treatment modalities (SRP + DL vs. SRP). Unlike traditional treatment methods using hand instruments, instrumentation of soft periodontal tissues using a DL $(980 \mathrm{~nm})$ resulted in complete removal of epithelial as demonstrated in Romanos, et al. through a pig model study [18]. The advantage of diode laser can be attributed to the bacterial effect it presents and might control the healing process [18].

The effectiveness of SRP alone and DL as an adjunct therapy to SRP in the treatment of periodontitis in a 6 month period was compared and evaluated in the clinical trial. Improvement in clinical parameters within the two cohorts is caused by removal of local etiological factors via mechanical debridement. Unlike SRP alone treated sites, there was statistically significant improvement in GI for SRP with adjunctive DL treated sites from baseline to 6,3 , and 1 month intervals. The credit for such beneficial improvements could be attributed to the advantage of using diode lasers as adjunctive therapy to SRP, which involve totally removing pocket epithelia [18]. Notably, the potential of adhering and invading periodontal epithelia is exhibited by periodontal pathogens that include $A$. actinomycetemcomitans and $P$. gingivalis [22-24]. In view of this, better formation of connective tissues is enabled by the essential role that the removal or elimination of periodontal pocket epithelia play in periodontitis treatment [18]. To avoid negative effects of systemic diseases and smoking on healing of periodontitis, non-smokers and systemically healthy people were chosen $[25,26]$.

The two treatment protocols offered satisfactory 
outcomes concerning the efficacy in periodontitis treatment and was concurrent with the findings of past studies, which indicated some successful outcomes with SRP alone and DL as adjunctive therapy to SRP [27].

There were no significant variations between DL + SRP and SRP alone cohorts at baseline. In the two treatment modalities applied, the results from this study were predictable and positive. There was significant improvement for both cohorts investigated at 6, 3, and 1 months from baseline with respect to clinical parameters.

The divergence and controversy of related studies regarding the application of laser therapy as the adjunct to traditional mechanical treatment for periodontal diseases as recommended and suggested by other studies formed the rationale of the present study. Safety and effectiveness of the laser was considered the main concern. For application in periodontitis treatment, other characteristics regarding $\mathrm{DL}$ were taken into account.

Consistent with the results of this study, clinical parameters did not improve when $810 \mathrm{~nm}$ DL combined with SRP was used. Nevertheless, the treatment required further costs and it was time consuming for dentist and patient. The two cohorts yielded positive outcomes following treatment; however, in terms of $\mathrm{Gl}$, better results were obtained from the test cohort. Notably, $\mathrm{PI}, \mathrm{CAL}$, and PD scores did not have differences. These results are similar to those that Borrajo, et al. obtained, which showed that the two cohorts did not differ [28]. Nevertheless, Borrajo, et al. reported that when $2 \mathrm{w}$ DL and $980 \mathrm{~nm}$ were used, significant differences existed in BOP [28]. Additionally, the findings of the study replicate those of Kreisler, et al. who examined clinical efficacy for DL as the adjunctive therapy to SRP within periodontitis treatment ( $1 \mathrm{~W}$ for 20 seconds, $810 \mathrm{~nm}$ diode laser) [11]. There were no significant variations for threemonth follow-ups. The findings from this study were consistent with those of Sgolastra, et al. meta-analysis, which summarized that DL as the adjunct to SRP did not offer more benefits with regard to improving clinical parameters [19]. Additionally, the findings of the study are aligned with those of Duki, et al. nevertheless, PD reduced significantly [29].

Our clinical outcomes were not consistent with those of Roncati and Gariffo's meta-analysis and systematic review, which showed that use of $\mathrm{DL}$ as an adjunct to SRP might present certain clinical benefits as opposed to SRP only for 6-month studies [30]. Furthermore, there is inconsistency in our findings with those of Slot, et al. meta-analyses and systematic review, which concluded that $\mathrm{DL}$ as an adjunct is considered moderate for CAL and PD changes with results indicating a negligible but considerable effect in support of diode laser [31].

Moreover, Salgam, et al. reported that sites treated with DL had variations in clinical parameters [17]. Qadri, et al. reported that unlike conventional treatment, GI, PI, and PD showed variations for the laser cohort [32]. Another study indicated that further treatment using diode laser might result in clinical parameters improving slightly for 6-month follow-up [8]. Besides, the results of an RCT indicated that there was an improvement for BOP index in $96.9 \%$ within the laser cohort, whereas in the control cohort it was a paltry $66.7 \%$. Unlike in control cohort, the laser cohort could witness a significant reduction in PD [6]. Varying application power density and wavelengths could account for such controversial findings.

AAP Best Evidence Consensus report (AAP BEC), reported lack of enough evidence regarding use of $D L$ as an adjunct in periodontitis treatment as acknowledged in clinical practice guidelines and broad conclusions cannot be supported because the overall evidence body quality is insufficient and there are few well-designed clinical studies [33]. The lack of improvement of PI, CAL, and PD for the present study could be attributed to the use of pulsed laser mode which could decrease laser light power density [34]; additionally, In our study we used irradiation wavelength of $810 \mathrm{~nm}$ and $2 \mathrm{~W}$ power which differ from previous studies which used $980 \mathrm{~nm}$ wavelength and noticed improved clinical parameters $[7,28,29]$. The dynamic wave mode can cause adjacent and target tissues to experience collateral damage when heat accumulates [34].

In regard to DL fiber diameter, 2 studies recorded improved clinical parameters by using a fiber diameter of $300 \mu \mathrm{m}[18,29]$. However, Prinat, et al. posited that deeper sockets could be accessed easily with smaller diameters [34], clinical parameters improved for one study that used $2000 \mu \mathrm{m}$ [28]. In the present study, a diameter of $400 \mu$ that lies between the 2 diameters used.

According to Prinat, 1-2 minutes for each tooth site (20-30 seconds per socket) is the ideal treatment time [34]. In reference to a previous study that used 30 seconds, clinical parameters showed improvement [7], whereas for other studies, there was no improvement [8]. Notably, clinical parameters improved for a study that irradiated periodontal pockets for 20 seconds using several applications [29]. All registered periodontal variables had an improvement in healing for a study by Üstün, et al. where $810 \mathrm{~nm}$ DL was used for irradiating 29 pockets for 20 seconds/site [35]. Additionally, in a study by Matarese, et al. DL 810 was used for 20 seconds/tooth, significant improvement was reported for the values of CAL and PD probing depth in DL + SRP as opposed to SRP only [36]. With the exception of $\mathrm{Gl}$, there were no significant variations between both cohorts even when 20 seconds were used in the study.

However, periodontal therapy led to increased patient time and cost when laser was added, thus, based on the few advantages demonstrated in this study, conventional treatment could be more cost-effective than it. 
There is need for further research to examine the effect of multiple uses of diode lasers on periodontal health.

\section{Conclusions}

Based on the measurement of clinical parameters CAL, PD, PI, and $\mathrm{GI}$, it could be concluded that the two modalities of treatment used might attain similar outcomes. Unlike SRP that is affordable, huge financial costs associated with advanced technology are required for laser therapy. There is a need for more diode-laserdriven periodontal therapy for adequately testing the potential benefits of controlled, randomized, and longitudinal clinical trials.

\section{Financial Support}

This study was funded by Jordan University of Science and Technology research grant.

\section{References}

1. Papapanou PN, Sanz M, Buduneli N, Dietrich T, Feres M, et al. (2018) Periodontitis: Consensus report of workgroup 2 of the 2017 World Workshop on the classification of periodontal and peri-implant diseases and conditions. J Periodontol 89: S173-S182.

2. Heitz-Mayfield LJ, Lang NP (2000) Surgical and nonsurgical periodontal therapy. Learned and unlearned concepts. Periodontol 62: 218-231.

3. Smiley CJ, Tracy SL, Abt E, Michalowicz BS, John MT, et al. (2015) Systematic review and meta-analysis on the nonsurgical treatment of chronic periodontitis by means of scaling and root planing with or without adjuncts. JADA 146: 508-524.

4. Schwarz F, Aoki A, Becker J, Sculean A (2008) Laser application in non-surgical periodontal therapy: A systematic review. J Clin Periodontol 35: 29-44.

5. Chanthaboury R, Irinakis T (2005) The use of lasers for periodontal debridement: Marketing tool or proven therapy? J Can Dent Assoc 71: 653-658.

6. Moritz A, Schoop U, Goharkhay K, Schauer P, Doertbudak O, et al. (1998) Treatment of periodontal pockets with a diode laser. Lasers Surg Med 22: 302-311.

7. Kamma JJ, Vasdekis VG, Romanos GE (2009) The effect of diode laser $(980 \mathrm{~nm})$ treatment on aggressive periodontitis: Evaluation of microbial and clinical parameters. Photomed Laser Surg 27: 11-19.

8. Caruso U, Nastri L, Piccolomini R, d'Ercole S, Mazza C, et al. (2008) Use of diode laser $980 \mathrm{~nm}$ as adjunctive therapy in the treatment of chronic periodontitis. A randomized controlled clinical trial. New Microbiol 31: 513-518.

9. Chambrone L, Ramos UD, Reynolds MA (2018) Infrared lasers for the treatment of moderate to severe periodontitis: An American Academy of Periodontology best evidence review. J Periodontol 89: 743-765.]

10. Dörtbudak O, Haas R, Bernhart T, Mailath-Pokorny G (2001) Lethal photosensitization for decontamination of implant surfaces in the treatment of peri-implantitis. Clin Oral Implants Res 12: 104-108.

11. Matthias K, Al Haj H, d'Hoedt B (2005) Clinical efficacy of semiconductor laser application as an adjunct to conventional scaling and root planing. Lasers Surg Med 37: 350-355.

12. De Micheli G, de Andrade AK, Alves VT, Seto M, Pannuti CM, et al. (2011) Efficacy of high intensity diode laser as an adjunct to non-surgical periodontal treatment: A randomized controlled trial. Lasers Med Sci 26: 43-48.

13. Schindl M, Kerschan K, Schindl A, Schön H, Heinzl H, et al. (1999) Induction of complete wound healing in recalcitrant ulcers by low-intensity laser irradiation depends on ulcer cause and size. Photodermatol Photoimmunol Photomed 15: 18-21.

14. Bjordal JM, Couppé C, Chow RT, Tunér J, Ljunggren EA (2003) A systematic review of low level laser therapy with location-specific doses for pain from chronic joint disorders. Aust J Physiother 49: 107-116.

15. Bjordal JM, Johnson MI, Iversen V, Aimbire F, LopesMartins RA (2006) Low-level laser therapy in acute pain: A systematic review of possible mechanisms of action and clinical effects in randomized placebo-controlled trials. Photomed Laser Surg 24: 158-168.

16. Andreas M, Ulrich S, Kawe G, Petra S, Orhun D, et al. (1997) Bacterial reduction in periodontal pockets through irradiation with a diode laser: A pilot study. J Clin Laser Med Surg 15: 33-37.

17. Saglam M, Kantarci A, Dundar N, Hakki S (2014) Clinical and biochemical effects of diode laser as an adjunct to nonsurgical treatment of chronic periodontitis: A randomized, controlled clinical trial. Lasers Med Sci 29: 3746.

18. Romanos George E, Henze M, Banihashemi S, Parsanejad $\mathrm{H}$, Winckler J, et al. (2004) Removal of epithelium in periodontal pockets following diode $(980 \mathrm{~nm})$ laser application in the animal model: An in vitro study. Photomed Laser Surg 22: 177-183.

19. Sgolastra F, Severino M, Gatto R, Monaco A (2013) Effectiveness of diode laser as adjunctive therapy to scaling root planning in the treatment of chronic periodontitis: A meta-analysis. Lasers Med Sci 28: 1393-1402.

20. Löe Harald, John S (1963) Periodontal disease in pregnancy I. Prevalence and severity. Acta Odontol Scand 21: 533-551:

21. Cobb CM, Low SB, Coluzzi DJ (2010) Lasers and the treatment of chronic periodontitis. Dent Clin North Am 54: 35-53?

22. Dierickx K, Pauwels M, Laine ML, Van Eldere J, Cassiman $\mathrm{JJ}$, et al. (2003) Adhesion of Porphyromonas gingivalis serotypes to pocket epithelium. J Periodontol 74: 844-848.

23. Papaioannou W, van Steenberghe D, Cassiman JJ, Dierickx K, Quirynen M (2003) Adhesion of porphyromonas gingivalis to cultured pocket epithelium: Mono-and multilayered. Clinical Oral Investigations 7: 162-166.

24. Mínguez $M$, Pousa $X$, Herrera D, Blasi $A$, Sánchez MC, et al. (2014) Characterization and serotype distribution of Aggregatibacter actinomycetemcomitans isolated from a population of periodontitis patients in Spain. Arch Oral Biol 59: 1359-1367.

25. Tonetti MS, Prato GP, Cortellini P (1996) Factors affecting the healing response of intrabony defects following guided tissue regeneration and access flap surgery. $\mathrm{J}$ Clin Periodontol 23: 548-556.

26. Trombelli L, Kim CK, Zimmerman GJ, Wikesjö UM (1997) Retrospective analysis of factors related to clinical outcome 
of guided tissue regeneration procedures in intrabony defects. J Clin Periodontol 24: 366-371.

27. Qadri T, Miranda L, Tuner J, Gustafsson A (2005) The short-term effects of low-level lasers as adjunct therapy in the treatment of periodontal inflammation. J Clin Periodontol 32: $714-719$.

28. Borrajo JL, Varela LG, Castro GL, Rodríguez-Nuñez I, Torreira MG (2004) Diode laser $(980 \mathrm{~nm})$ as adjunct to scaling and root planing. Photomed Laser Surg 22: 509512.

29. Dukić W, Bago I, Aurer A, Roguljić M (2013) Clinical effectiveness of diode laser therapy as an adjunct to nonsurgical periodontal treatment: A randomized clinical study. J Periodontol 84: 1111-1117.

30. Roncati M, Gariffo A (2014) Systematic review of the adjunctive use of diode and Nd:YAG lasers for nonsurgical periodontal instrumentation. Photomed Laser Surg 32: 186197.

31. Slot DE, Jorritsma $\mathrm{KH}$, Cobb CM, Van der Weijden FA (2014) The effect of the thermal diode laser (wavelength 808-980 nm) in non-surgical periodontal therapy: A systematic review and meta-analysis. J Clin Periodontol 41: 681-692.
32. Qadri T, Tunér J, Gustafsson A (2015) Significance of scaling and root planing with and without adjunctive use of a water-cooled pulsed Nd: YAG laser for the treatment of periodontal inflammation. Lasers Med Sci 30: 797-800.

33. Mills MP, Rosen PS, Chambrone L, Greenwell H, Kao RT, et al. (2018) American Academy of Periodontology best evidence consensus statement on the efficacy of laser therapy used alone or as an adjunct to non-surgical and surgical treatment of periodontitis and peri-implant diseases. J Periodontol 89: 737-742.]

34. Pirnat $S$ (2007) Versatility of an $810 \mathrm{~nm}$ diode laser in dentistry: An overview. JLHA 4: 1-9.

35. Üstün K, Erciyas K, Sezer U, Şenyurt SZ, Gündoğar H, et al. (2014) Clinical and biochemical effects of $810 \mathrm{~nm}$ diode laser as an adjunct to periodontal therapy: A randomized split-mouth clinical trial. Photomed Laser Surg 32: 61-66.

36. Matarese G, Ramaglia L, Cicciù M, Cordasco G, Isola G (2017) The effects of diode laser therapy as an adjunct to scaling and root planing in the treatment of aggressive periodontitis: A 1-year randomized controlled clinical trial. Photomed Laser Surg 35: 702-709. 